Rufina M. Tretyakova, Andreas Meyerhans, and Gennady A. Bocharov*

\title{
A drug pharmacodynamics and pharmacokinetics based approach towards stabilization of HIV infection dynamics
}

\begin{abstract}
In our study we developed a computational algorithm for finding optimal dosages of antiretroviral drug administration for the stabilization of HIV load at low levels. The novelty is that the pharmacokinetics and pharmacodynamics of the antiretroviral drugs were taken into account. A standard closed-loop control of HIV dynamics was constructed that stabilizes the viral load and the optimal drug administration mode was formulated. We analyze the appropriateness of the 'drug efficacy' based control and its relationship to a realistic drug dosage and kinetic models in the human body. The translation of the efficacy function into drug concentration is implemented via the pharmacodynamics model of the drug effect. Optimal approximation of the idealized concentration functions is based on the orthogonal projection on linear subspace of drug pharmacokinetics functions.
\end{abstract}

Keywords: HIV dynamics, stabilization, feedback control, pharmacodynamics, pharmacokinetics.

MSC 2010: 49J15, 49N90, 95B05, 92C50

DOI: $10.1515 /$ rnam-2015-0027

Received July 17, 2015; accepted August 25, 2015

Human immunodeficiency virus (HIV) infections remain a major concern for public health with about 35 million infected individuals worldwide [10]. HIV is a retrovirus that affects the immune system, and uses its functionality for self-replication. Continuous ongoing virus replication within an infected host leads to a gradual destruction of the immune system and results in AIDS and eventual death. Antiretroviral therapy (ART) based on the use of a combination of three and more pharmaceutical compounds out of the 26 approved drugs provides a basis for long-term control of virus replication $[3,19]$. The drugs target different steps of the viral life cycle and allow to keep viral loads at low level. This transforms HIV-infected individuals into chronic carriers with prolonged life expectancy. However, ART is rather expensive and associated with side effects such as hepatotoxicity, neurological disorders and cardiovascular diseases. Therefore, the optimal timing of medication presents an important issue with respect to improving therapy benefits.

Mathematical modelling and control theory represent promising tools for developing parsimonious and personalized approaches to treatment of HIV-infected individuals. An overview of the progress achieved so far in model-driven analysis and optimal control of HIV infection can be found in $[1,5,6,8,13]$. As the virus cannot be eliminated via antiretroviral therapy, the realistic objective in formulating the optimal control problem is the reduction of the viral load. Indeed, the duration of an asymptomatic HIV infection is inversely related to the set-point viral load as shown in [7]. The appropriate framework for designing the corresponding control strategy is based upon the feedback and deviation control [4, 14]. The antiretroviral drugs are given to patients according to the manufacturer's recommendation. It should be noted that research on optimal timing of ART presents a challenge. Drug dynamics in the body is governed by drug-specific pharmacokinetic models with respective parameters $[11,18,20]$. However, the existing approaches towards the mathematical modelling of optimal therapy design are based upon the so called 'drug efficacy' functions or control variables that can

Rufina M. Tretyakova: Institute of Numerical Mathematics of the RAS, Moscow 119333, Russia. Lomonosov Moscow State University, Moscow 119991, Russia

Andreas Meyerhans: Infection Biology Group, Department of Experimental and Health Sciences, University Pompeu Fabra, Barcelona 08003, Spain. Institucio Catalana de Recerca i Estudis Avancats (ICREA), Barcelona 08010, Spain

*Corresponding Author: Gennady A. Bocharov: Institute of Numerical Mathematics of the RAS, Moscow 119333, Russia. E-mail: bocharov@inm.ras.ru 
take any values from the specified range $[1,6,8,14]$ and thus ignore drug kinetic constraints. We note that in [8] an optimal control problem for three drugs treatment was considered with the drug concentrations in between the dosages administration approximated as average values rather than dynamic quantities.

The purpose of our study was to develop a numerical method for finding an optimal dosage of medication for HIV control taking into account the pharmacokinetics and pharmacodynamics of the antiretroviral drugs. We constructed a closed-loop control of HIV dynamics that stabilizes the viral load and evaluated the optimal drug administration regime. We analyzed the appropriateness of the 'drug efficacy' based control and its relationship to the drug dosage and kinetics in the body. Our study is built upon the previous work on closed-loop control [14]. In Section 2 we present the basic model of HIV dynamics and the feedback-based stabilization procedure. Section 3 considers the pharmacokinetic and pharmacodynamic issues for drug modelling and the algorithm for optimal drug dosage calculation. The numerical implementation and the results are covered in Section 4. Finally, in Section 5 the conclusions are presented.

\section{Basic approach to computational modelling of HIV infection stabilization}

We use the following notation in the paper:

- $\quad T(t)$ density of uninfected $\mathrm{CD} 4^{+} \mathrm{T}$-cells at time $t$;

- $T^{*}(t)$ density of infected CD4 $4^{+}$T-cells at time $t$;

- $V(t)$ concentration of free virions (HIV RNA particles) at time $t$;

- $\quad e(t)$ drug efficacy (bounded value representing relative effect of HIV treatment) at time $t$;

- $e_{\text {max }}$ maximal drug efficacy;

- $C(t)$ drug concentration at time $t$;

- $\quad C_{0}$ drug dosage (amount of administered drug divided by the peripheral blood volume in which it is distributed);

- RTI reverse transcriptase inhibitors (antiviral drug inhibiting the transcription of the viral single stranded RNA genome into a double-stranded viral DNA);

- PI protease inhibitors (antiviral drug blocking the production of infectious viral particles).

The overall HIV treatment process can be described as follows: the patient takes medication regularly to sustain the drug concentration $C(t)$ around some target value $\left(C(t) \simeq C_{0}\right)$. The drug concentration determines the drug efficacy $e(t) \in\left[0, e_{\max }\right)$, that eventually determines the reduction of the virus population growth $V(t)$. A conventional approach to modelling HIV treatment is based on predicting the viral population dynamics $V(t)$ given the drug concentration $C(t)$ and the efficacy $e(t)$ function (see, e.g. [20]). The application of the optimal control methods to HIV infection dynamics consists in computing the optimal efficacy function $e(t)$ in order to minimize some infection severity criterion depending on viral load $V(t)$, immune status and drug quantity (see, e.g. [1, 8]).

\subsection{Basic model of HIV infection dynamics}

Today, there exists a broad spectrum of mathematical models addressing various aspects of the HIV infection dynamics and formulated with ordinary, delay- and integro-differential equations (see the references in [5]). Most of the research on optimal control of HIV infection dynamics has been performed with rather simple mathematical models considering the target cell infection and the immune response (see Tables 1-2 in [6]). The widely accepted model used in the studies of the HIV infection is the one presented in [13]. It will be used for illustrative purpose in the present study. We note, that more complex models have been developed that consider different types of immune system cells and their characteristic features, infection latency, and virus 
mutation (see [1, 8, 12]). In the following analysis we consider the HIV dynamics model presented in [13] and used for feedback control study in [14]. Model variables represent the uninfected target cells $T(t)$ [cell $/ \mathrm{mm}^{3}$ ], the productively infected cells $T^{\star}(t)$ [cell $\left./ \mathrm{mm}^{3}\right]$ and the virions $V(t)$ [copies $/ \mathrm{mm}^{3}$ ]. The model considers two drugs: RTI drug reducing the infectivity rate, and PI drug inhibiting the virion production by the infected cells. The studies of multiple drug administration [18] proved that their effects can be considered independent:

$$
\left\{\begin{array}{l}
\frac{\mathrm{d} T(t)}{\mathrm{d} t}=-\mu_{1} T(t)-\left(1-e_{R T I}(t)\right) \beta T(t) V(t)+s \\
\frac{\mathrm{d} T^{\star}(t)}{\mathrm{d} t}=-\mu_{2} T^{\star}(t)+\left(1-e_{R T I}(t)\right) \beta T(t) V(t) \\
\frac{\mathrm{d} V(t)}{\mathrm{d} t}=-\mu_{3} V(t)+\left(1-e_{P I}(t)\right) k T^{\star}(t) .
\end{array}\right.
$$

We use the same parameter values as in [14]: $\mu_{1}=0.02$ day $^{-1}, \mu_{2}=2.4$ day $^{-1}, \mu_{3}=0.24$ day $^{-1}$ are healthy T-cells, infected T-cells and virions death rates, respectively; $s=10\left(\right.$ cell $/\left(\mathrm{mm}^{3} \cdot\right.$ day $\left.)\right)-$ constant source of $\mathrm{CD}^{+} \mathrm{T}$-cells (produced by thymus); $\beta=2.4 \times 10^{-5}\left(\mathrm{~mm}^{3} /\right.$ (virion $\cdot$ day)) - infectivity rate of free virions; $k=100$ (virion/(cell $\cdot$ day)) - virion production rate by infected cells.

\subsection{Stabilization of HIV dynamics}

The implemented algorithm of computing the closed-loop control is based on the work [14]. First, we rewrite system (1.1) using the following vector variables, $x, u$ :

$$
x(t)=\left[\begin{array}{lll}
T(t) & T^{\star}(t) & V(t)
\end{array}\right], \quad u(t)=\left[\begin{array}{ll}
\frac{e_{R T I}(t)}{e_{\max }} & \frac{e_{P I}(t)}{e_{\max }}
\end{array}\right] \Rightarrow \frac{\mathrm{d} x(t)}{\mathrm{d} t}=f(x(t), u(t)) .
$$

Vector $x(t)$ states HIV dynamics variables and vector $u(t)$ represents control variables for efficacy functions which are normalized to the half-open interval $[0,1)$. The aim of HIV infected patient ART based optimal control is not a complete elimination of the virus, but the maintenance of its concentration (viral load) at a constant low level. Therefore, the system is to be stabilized in the vicinity of a low viral load equilibrium point $X^{s s}$, which can be found by setting derivative to zero $(\dot{x}(t)=0)$. The steady state system of equations reads:

$$
\left\{\begin{array}{l}
x_{2}^{s s}=\left(s-\mu_{1} x_{1}^{s S}\right) / \mu_{2} \\
u_{1}^{s S}=1-\mu_{2} x_{2}^{s S} /\left(\beta x_{1}^{s S} x_{3}^{s S}\right) \\
u_{2}^{s s}=1-\mu_{3} x_{3}^{s S} /\left(k x_{2}^{s S}\right) .
\end{array}\right.
$$

There are three equations with five unknown variables, which means that two of them can be taken as independent variables while the other three are expressed through them. Similar to [14], we make use the U.S. Department of Health and Human Services HIV Therapy Guidelines [16] postulating that the number of virions should be suppressed under $50 \mathrm{~mm}^{-3}$. We set $x_{1}^{s S}=490$ and $x_{3}^{s S}=30$. In order to keep $x_{1}^{s s}$ on a high level we need $u_{j}^{s s} \simeq 1$, which is practically unrealistic. From the above formulas we obtain:

$$
x^{s s}=\left[\begin{array}{lll}
490 & 0.83 & 30
\end{array}\right], \quad u^{s s}=\left[\begin{array}{ll}
0.43 & 0.14
\end{array}\right] .
$$

The asymptotic stability of the equilibrium point $\left(x^{s s}, u^{s s}\right)$ can be examined via the analysis of the eigenvalues of Jacobian matrix generating the following characteristic polynomial:

$$
\left(\lambda+\mu_{1}+\left(1-u_{1}^{s S}\right) \beta x_{3}^{S S}\right)\left(\lambda+\mu_{2}\right)\left(\lambda+\mu_{3}\right)-k \beta\left(1-u_{1}^{s S}\right)\left(1-u_{2}^{s S}\right) x_{1}^{S S}\left(\lambda+\mu_{1}\right)=0 .
$$

It can be verified that the above steady state is stable.

The control problem of stabilizing the system at the steady state can be formulated in terms of the deviations $\Delta x(t)$ from constant equilibrium values $x^{s s}$. The control variables also can be splitted into the constant component $u^{s s}$ and time-variant component $\Delta u(t)$ :

$$
x_{i}(t)=x_{i}^{s S}+\Delta x_{i}(t), \quad u_{j}(t)=u_{j}^{s S}+\Delta u_{j}(t) .
$$


The state and control variables are related via the linearized system of equations:

$$
\begin{gathered}
\dot{x}(t)=(\dot{\Delta x})(t)=f(x(t), \quad u(t)) \simeq A \Delta x(t)+B \Delta u(t) \\
A=\left[\begin{array}{ccc}
-\mu_{1}-\left(1-u_{1}^{s s}\right) \beta x_{3}^{s s} & 0 & -\left(1-u_{1}^{s s}\right) \beta x_{1}^{s s} \\
\left(1-u_{1}^{s s}\right) \beta x_{3}^{s S} & -\mu_{2} & \left(1-u_{1}^{s S}\right) \beta x_{1}^{s s} \\
0 & \left(1-u_{2}^{s s}\right) k & -\mu_{3}
\end{array}\right], \quad B=\left[\begin{array}{cc}
\beta x_{1}^{s s} x_{3}^{s s} & 0 \\
-\beta x_{1}^{s s} x_{3}^{s s} & 0 \\
0 & -k x_{2}^{s s}
\end{array}\right] .
\end{gathered}
$$

We consider the quadratic performance criterion to obtain $\Delta u(t)$ :

$$
\left.Q(\Delta x, \Delta u)=\int_{0}^{\infty} \Delta x^{T}(t) W_{1} \Delta x(t)+\Delta u^{T}(t) W_{2} \Delta u(t)\right) \mathrm{d} t
$$

where $W_{1}>0$ and $W_{2}>0$ are some positively-defined weighting matrices. It is known that the optimal control law that minimizes the above performance criterion along the trajectories of linearized equation can be calculated from matrix Riccati equation [2]:

$$
\begin{gathered}
\Delta u^{\mathrm{opt}}(t)=-F^{\mathrm{opt}} \Delta x(t), \quad F^{\mathrm{opt}}=W_{2}^{-1} B^{T} P \\
P A+A^{T} P+W_{1}-P B W_{2}^{-1} B^{T} P=0 .
\end{gathered}
$$

\section{Pharmacokinetics and pharmacodynamics based stabilization of the infection}

To compute the optimal drug dosages which allow to achieve the drug efficacy required by the stabilizing control function $u(t)=u_{s s}+\Delta u(t)$, we consider the standard models of pharmacokinetics and pharmacodynamics [11]. Combined pharmacokinetic-dynamic studies seek to characterize the time course of drug effects.

\subsection{Pharmacodynamics}

Pharmacodynamics studies the mechanism of interaction between drug and cellular receptors as well as the drug transformation in the cells. Pharmacodynamic models exclusively relate the drug concentration with the pharmacological effect $e(t)=e(t, C(t))$. Pharmacodynamics is linked to pharmacokinetics, which encompasses the study of movement of drugs into, through, and out of the body. For any given efficacy function $e(t)$ one can compute the corresponding drug concentration $C(t)$. The basic pharmacodynamic models are presented in Table 1. The selection of an appropriate pharmacodynamic model depends on drug characteristics as well as the way of its administration.

The pharmacodynamic model parameters can be estimated from experimental and clinical data as presented in [20]. The parameter values used in our paper represent some reference values taken from [11] for illustrative purpose. The inversion of the drug efficacy function into the drug concentration profile is given by the parameterization specified in the last column of the above Table. We note that whereas for the sigmoid and time-variant models the concentration function exists for any efficacy function $e(t)$, for the irreversible and indirect link models the differentiability of $e(t)$ is required.

\subsection{Pharmacokinetics}

The subject of pharmacokinetics is the study of the spatial-temporal behaviour of the drug concentration in the organism [17]. Pharmacokinetic models suggest explicit functions of drug concentration $C(t)=C\left(t, C_{0}, \tau\right)$, where $\tau$ is a time interval between drug administration (e.g., $\tau=\{2,4,6,8,12,24, \ldots\}$ hours) and $C_{0}=$ 
Table 1. Basic pharmacodynamic models: efficacy function $e(C(t))$ and the inverse concentration functions $C(e(t))$.

\begin{tabular}{|c|c|c|c|}
\hline Model & Description & $e(C)$ & $C(e), \mathrm{ng} \cdot \mathrm{mm}^{-3}, \mathrm{day}^{-1}[15]$ \\
\hline Sigmoid $e_{\max }$ & $\begin{array}{l}\text { based on occupancy theory and } \\
\text { used if chemical equilibrium is } \\
\text { achieved }\end{array}$ & $\begin{array}{l}e(t)=\frac{e_{\max } C(t)}{I C_{50}+C(t)}, \\
I C_{50}-\text { concentration at } \\
\text { half-maximal effect }\end{array}$ & $C(t)=I C_{50} \frac{e(t)}{e_{\max }-e(t)}, I C_{50}=8.0$ \\
\hline Indirect link & $\begin{array}{l}\text { used in case of temporal } \\
\text { dissociation between time courses } \\
\text { of concentration and effect }\end{array}$ & $\begin{array}{l}e(t)=\frac{e_{\max } y(t)}{l y_{50}+y(t)} \\
y(t)=\int_{0}^{t} C(\tau) e^{-k_{y}(t-\tau)} d \tau \\
y(t)-\text { concentration in receptor } \\
\text { site proximity, } k_{y} \text { - delay constant }\end{array}$ & $\begin{array}{l}C(t)=\frac{\dot{e}(t) / y_{50}}{e_{\max }-e(t)}\left(k_{y} e(t)+\frac{e_{\max }}{e_{\max }-e(t)}\right) \\
k_{y}=0.8, I y_{50}=10.0\end{array}$ \\
\hline Irreversible & $\begin{array}{l}\text { used if drug-receptor bimolecular } \\
\text { interaction is irreversible }\end{array}$ & $\begin{array}{l}\dot{e}(t)=r(e(t))-g(C) e(t) \\
r(e)-\text { proliferation function, } \\
g(C) \text { - cell-killing function }\end{array}$ & $\begin{array}{l}C(t)=g^{-1}\left(\frac{r(e)-\dot{e}}{e(t)}\right), r(e)=k_{\text {in }}-k_{\text {out }} e \\
k_{\text {in }}=0.5, k_{\text {out }}=1.0, g(C)=C\end{array}$ \\
\hline Time-variant & $\begin{array}{l}\text { used if drug susceptibility is } \\
\text { nonconstant }\end{array}$ & $\begin{array}{l}e(t)=\frac{e_{\max } C(t)}{I C_{50}(t)+C(t)} \\
I C_{50}(t)-\text { time course of } \\
\text { concentration required for } \\
\text { half-maximal effect }\end{array}$ & $\begin{array}{l}C(t)=I C_{50}(t) \frac{e(t)}{e_{\max }-E(t)} \\
I C_{50}(t)=I_{t 0}+\left(I_{t n}-I_{t 0}\right) t / T_{\text {cure }} \\
I_{t 0}=7.0, I_{t n}=8.5\end{array}$ \\
\hline
\end{tabular}

Table 2. Basic pharmacokinetic models: the drug concentration functions $C(t)$.

\begin{tabular}{|c|c|c|c|}
\hline Model & Description & $C(t)$ & Parameters, day ${ }^{-1}[16]$ \\
\hline One-compartment (1C) & $\begin{array}{l}\text { concentration decreases } \\
\text { exponentially }\end{array}$ & $C(t)=C_{0} e^{-\alpha t}$ & $\alpha=2.33$ \\
\hline $\begin{array}{l}\text { One-compartment with } \\
\text { absorption (1CA) }\end{array}$ & $\begin{array}{l}\text { drug absorption from gastric } \\
\text { system is considered }\end{array}$ & $C(t)=C_{0}\left(e^{-\alpha t}-e^{-k_{1} t}\right), k_{1}>\alpha$ & $\alpha=3, k_{1}=9$ \\
\hline Two-compartment (2C) & $\begin{array}{l}\text { second compartment is a } \\
\text { drug reservoir e.g. blood and } \\
\text { cellular plasma }\end{array}$ & $C(t)=C_{0}\left(a_{1} e^{-\alpha t}+a_{2} e^{-\beta t}\right)$ & $\begin{array}{l}\alpha=3.4, \beta=0.9, a_{1}=0.33 \\
a_{2}=0.66\end{array}$ \\
\hline $\begin{array}{l}\text { Two-compartment with } \\
\text { absorption ( } 2 \mathrm{CA})\end{array}$ & unites all the previous & $\begin{array}{l}C(t)= \\
C_{0}\left(a_{1} e^{-\alpha t}+a_{2} e^{-\beta t}-a_{0} e^{-k_{1} t}\right) \\
k_{1}>\alpha, k_{1}>\beta\end{array}$ & $\begin{array}{l}\alpha=1.9, \beta=3, k_{1}=16 \\
a_{1}=a_{2}=0.25, a_{0}=0.5\end{array}$ \\
\hline
\end{tabular}

$M_{0} / V$ is an initial dosage. The classical pharmacokinetic models $[11,17]$ are based on the concept of compartment: compartmental system is made up of a finite number of constant volume compartments, each of which is homogeneous and well mixed; compartments interact by exchanging material. The basic drug kinetic functions for different compartmental models are presented in Table 2. The identification of an appropriate model for a specific drug requires the drug concentration profiles and is beyond the scope of our study. The parameter values specified in Table 2 are for illustrative purpose taken from [17]).

\subsection{Pharmacokinetic-dynamic model based approximation of control function}

In this section we seek to find the best approximation of the feedback control law using the drug concentration functions representing the drug pharmacokinetics in vivo. The continuous drug concentration function to be approximated $C(t), t \in\left[0, T_{\text {cure }}\right]$ is an element of Hilbert space $H=L_{2}\left(0, T_{\text {cure }}\right)$. The control (or drug efficacy) function $u(t) \equiv e(t)$ calculated from (1.2) and (1.1) is continuous [2], therefore the superposition of $u(t)$ and $C(u)$ (see Table 1$)$ is also continuous: $C \in L_{2}\left(0, T_{\text {cure }}\right)$. If the control function $u(t)$ is represented at the mesh points dividing the interval $\left[0, T_{\text {cure }}\right.$ ] into $M$ equal subintervals, then the vector $C \in \mathbb{R}^{M}$ and $H$ in this case is the $M$-dimensional Euclidean space. We use the following notation for the inner product $(\cdot, \cdot)=(\cdot, \cdot)_{H}$ and the norm $\|\cdot\|=\|\cdot\|_{H}$.

It is practically impossible to achieve an exact match between the theoretical (continuous optimal control function) and the actual drug concentration in blood, since the drug kinetics is governed by an appropriate pharmacokinetic model. If the drug is administered at dose $C_{0}$ at time $t=0$, its concentration changes as 
$C(t)=C_{0} \Gamma(t), t \in\left[0, T_{\text {cure }}\right]$ according to one of the models:

$$
\Gamma(t)=\left\{\begin{array}{ll}
\Gamma^{P K}(t), & t \geq 0 \\
0, & t<0,
\end{array} \quad \Gamma^{P K}(t)= \begin{cases}\mathrm{e}^{-\alpha t}, & \text { one-compartment } \\
\mathrm{e}^{-\alpha t}-\mathrm{e}^{-k_{1} t}, & \text { one-compartment with absorption } \\
a_{1} \mathrm{e}^{-\alpha t}+a_{2} \mathrm{e}^{-\beta t}, & \text { two-compartment } \\
a_{1} \mathrm{e}^{-\alpha t}+a_{2} \mathrm{e}^{-\beta t}-a_{0} \mathrm{e}^{-k_{1} t}, & \text { two-compartment with absorption. }\end{cases}\right.
$$

One can specify an appropriate pharmacokinetic model $\Gamma^{P K}$ by selecting one of above four kinetic pattern functions.

Let the time interval between drug administration be $\tau$ and define a uniform grid $t_{i}=i \tau, i=0, \ldots, N-1$, on $t \in\left[0, T_{\text {cure }}\right]$. The therapy period $T_{\text {cure }}$ is divided into intervals $\tau_{i}=\left[t_{i}, t_{i+1}\right]$ so that $\tau N=T_{\text {cure. }}$. If the drug is administered at dose $C_{0}^{i}$ at time $t_{i}$, its kinetics follows $C_{0}^{i} \Gamma\left(t-t_{i}\right)$. Consider a set of basis functions $\Gamma_{i}=\Gamma\left(t-t_{i}\right)$. Then, for any $t \in\left[0, T_{\text {cure }}\right]$ the drug concentration function is a linear combination of the basis functions as follows:

$$
C_{\Gamma}(t)=\sum_{i=0}^{N-1} C_{0}^{i} \Gamma_{i}
$$

Therefore, all admissible drug concentration functions $C_{\Gamma}(t)$ are the elements of a linear span of the basis functions $\left\{\Gamma_{i}\right\}_{i=0}^{N-1}$. Let us denote the finite linear subspace of $H, \mathcal{L}_{\Gamma}=\operatorname{span}\left(\Gamma_{i}\right), i=0, \ldots, N-1$. The optimal approximation problem in Hilbert space for given concentration function $C$ is equivalent to the minimization problem for the following functional:

$$
Z\left(C_{0}\right)=\left\|C(t)-\sum_{i=0}^{N} C_{0}^{i} \Gamma_{i}\right\| \rightarrow \inf , \quad C_{0}=\left[C_{0}^{1} \ldots C_{0}^{N}\right]^{T} \in \mathbb{R}^{N} .
$$

The following statement holds.

Theorem 2.1. The solution of the orthogonal projection problem (2.2) exists, is unique and given by $C_{0}^{\star}=G^{-1} \tilde{C}$, where $\tilde{C}$ is the drug profile function required for optimal feedback stabilization and $G$ is the Gram matrix of the basis functions $\left\{\Gamma_{i}\right\}_{i=0}^{N-1}$.

Proof. The Hilbert projection theorem [15] states that for any element of the Hilbert space and any its finite closed subspace there exists a unique best approximation element of that subspace. Therefore, $\forall C \in H$ there exists a unique orthogonal projection $C_{\Gamma}^{\star} \in \mathcal{L}_{\Gamma}$ such that:

$$
C-C_{\Gamma}^{\star} \perp \mathcal{L}_{\Gamma} \Rightarrow\left\|C-C_{\Gamma}^{\star}\right\|=\inf _{Y \in \mathcal{L}_{\Gamma}}\|C-Y(t)\| .
$$

To find $C_{\Gamma}^{\star}$, the orthogonal projection procedure $\left(C-C_{\Gamma}^{\star}, \Gamma_{i}\right)=0, i=0, \ldots, N-1$, generates the following system of linear algebraic equations $\tilde{C}_{i}=\left(C, \Gamma_{i}\right)$, depending on the Gramian matrix with elements $G_{i j}=$ $\left(\Gamma_{j}, \Gamma_{i}\right)$ for the basis functions $\mathcal{L}_{\Gamma}:\left(\sum_{j=0}^{N} C_{0}^{j} \Gamma_{j}, \Gamma_{i}\right)=\sum_{j=0}^{N} C_{0}^{j}\left(\Gamma_{j}, \Gamma_{i}\right)$. The best-fit estimate of the dosages vector $C_{0}^{\star}$ is given by the solution of the linear system $G C_{0}^{\star}=\tilde{C}$.

Another way to prove the theorem is to find the minimum to the functional $Z\left(C_{0}\right)$. At the extremum point the following holds: $(\nabla Z)_{i}=-2\left(C-C_{\Gamma}^{\star}, \Gamma_{i}\right)=0$ and the Hessian matrix of $Z\left(C_{0}^{*}\right)$ is $\left(Z^{\prime \prime}\right)_{i j}=2\left(\Gamma_{j}, \Gamma_{i}\right), Z^{\prime \prime}=$ $2^{N} G$. As the Hessian matrix is constant for all $C_{0} \in \mathbb{R}^{N}$, and is positive definite, it follows that $Z$ is a convex functional. Therefore, it has a unique minimum point defined by $\nabla Z=0$.

Overall, both considerations lead to the same system with a positive definite matrix for the unknown drug dosages $G C_{0}=\tilde{C}$ that has a unique solution $C_{0}^{\star}=G^{-1} \tilde{C}$.

Remark 2.1. The Gram matrix $G$ is a symmetric and Toeplitz one: $\left(\Gamma_{j}, \Gamma_{i}\right)=\left(\Gamma_{j}, \Gamma_{i}\right)=\left(\Gamma_{i+k}, \Gamma_{j+k}\right)$ for all $k: 0 \leq$ $i+k<N$ and $0 \leq j+k<N$, because all functions $\Gamma_{i}$ are just the shifts of the same function $\Gamma$. The solution of the system $G C_{0}=\tilde{C}$ with a symmetric Toeplitz matrix can be obtained by means of the Levinson recursion algorithm in $O\left(N^{2}\right)$ operations. 


\section{Numerical study}

\subsection{Implementation of the algorithm}

The following iterative process to compute the optimal drug dosages allowing to approximate the drug concentration profile required by the the feedback control law is implemented using MATLAB programming tools.

Initially, the control time interval is set $\left[0, T_{\text {cure }}\right]$. Then, a uniform mesh $t_{n}, n=1, \ldots, N$, is introduced with the step-size $h$, such that $t_{N}=h N=T_{\text {cure. }}$. On the set of subintervals $\left[t_{n-1}, t_{n}\right]$, the control function $u(t)$ and the trajectory $x(t)$ are computed using the following procedure:

(1) compute $\Delta u\left(t_{n-1}\right)=-F_{n-1} \Delta x\left(t_{n-1}\right)$, where the optimal gain matrix $F_{n-1}$ is computed by solving matrix Riccati equation (1.2);

(2) evaluate $u\left(t_{n-1}\right)=u_{s s}+\Delta u\left(t_{n-1}\right)$, with $u\left(t_{n-1}\right)$ normalized to $0 \leq u\left(t_{n-1}\right)<1$;

(3) solve the initial value problem $\dot{x}(t)=f(x(t), u(t))$ on $\left[t_{n}, t_{n-1}\right]$ starting at $x\left(t_{n-1}\right)$ with $u(t)=u\left(t_{n-1}\right)$ to get $u\left(t_{n}\right)$.

As the result we generate the (discrete) functions $x\left(t_{n}\right)$ converging to $x^{s s}$ and optimal drug efficacy functions $u\left(t_{n}\right) \rightarrow u_{s s}$, respectively.

The next stage is to compute the corresponding concentration profile $C(t)$ for the generated drug efficacy function $u\left(t_{n}\right)$ using the specific pharmacodynamic formula $C(u(t))$ as indicated in Table 1 . Finally, we solve the linear system $G C_{0}^{*}=\tilde{C}$ for $C_{0}^{*}$ considering different values of time intervals between the drug administration, characterized by the parameter $\tau=\{2,4,6,8,10,12,24\}$ hours. Overall, this allows one to estimate the best-fit drug dosages vector $C_{0}^{\star}$ for different $G(\tau)$ and $\tilde{C}(\tau)$.

\subsection{Results}

We consider the stabilization problem for the HIV infection with the initial conditions that differ from the target steady state values: $\left[T, T^{\star}, V\right]=[560,1,60]$. We set the duration of the therapy time $T_{\text {cure }}=250$ days and find the solution of the optimal feedback control problem on a uniform grid with a step $(h)$ of one hour $\left(t_{n}=n /\left(24 T_{\text {cure }}\right)\right)$. The controlled dynamics of the viral load $V(t, u(t))$, uninfected $T(t, u(t))$ and infected T-cells populations $T^{\star}(t, u(t))$ is shown in Fig. 1.

The closed-loop system is driven to the neighbourhood of the steady state $x_{s s}$. The corresponding control (efficacy) functions $u_{1}, u_{2}$ are presented in Fig. 2.

Notably, for this scenario, RTI-type drug is not required in the treatment aimed at the HIV infection stabilization of up to 120-th day, while the PI-type drug should be administered in large quantities: for the first ten days the patient is supposed to receive the drug amount that brings the efficacy function to almost 1 . Then it should be reduced by $50 \%$. After 120 days the required drug efficacy level goes down to 0.14 , whereas the effect of the first drug should ensure the efficacy function value of about 0.43 .

To examine the difference between the dynamic and static modes of control, we present the solution to the HIV model under the assumption that the control functions take constant values equal to the mean values of drug efficacy function $x(t, A U C(u(t)))$ (the mean value AUC is the area under function curve divided by therapy time, which are in our case $A U C\left(u_{1}\right)=0.216$ and $A U C\left(u_{2}\right)=0.336$ ). Figure 3 shows the optimally controlled and time constant control HIV infection dynamics. The differences in the time course of the state variables between the optimal control- and constant drug efficacy based therapy are quite substantial.

Using the computed efficacy functions, we proceed with the inversion of the required control laws into the drug medication levels using four pharmacodynamic models specified in Table 1. Figure 4 shows the kinetics of the drugs concentrations for the different models. The drug concentration functions are rather close to the efficacy function patterns (Fig. 2) although some smoothing of the sharp peak of the PI control law takes place. The resulting functions for indirect link- and irreversible models appear to be quite similar to the $e_{\max }$ model. The time-variant model demonstrates the presence of a small linear growth phase in RII drug after 120 


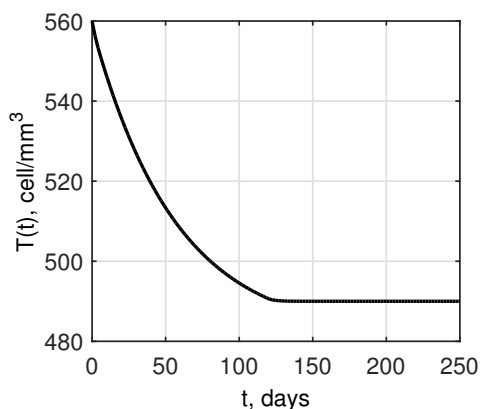

(a)

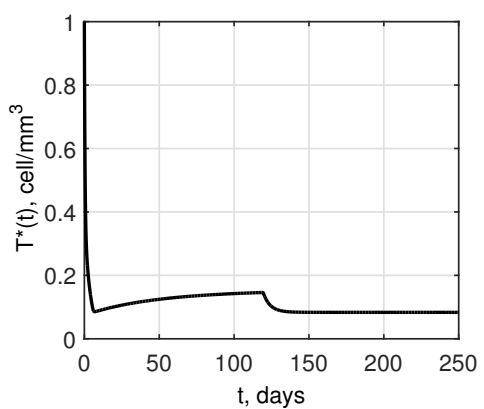

(b)

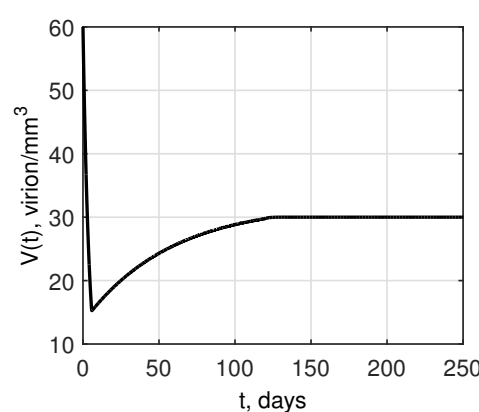

(c)

Figure 1. Stabilization of the HIV infection dynamics: (a) uninfected target CD4 ${ }^{+}$T-cells, (b) infected CD4 ${ }^{+}$T-cells and (c) viral load.

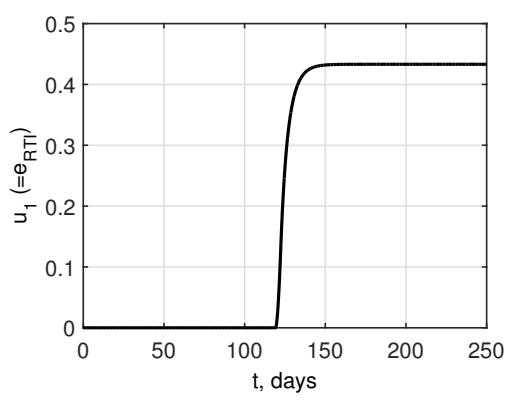

(a)

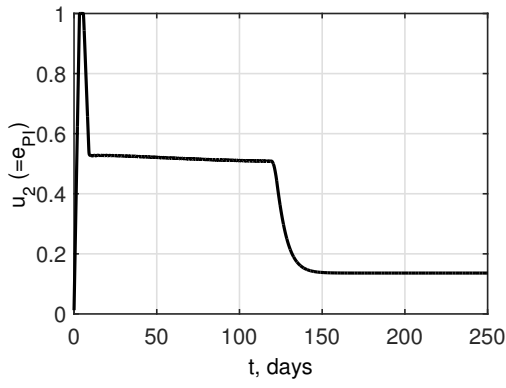

(b)

Figure 2. The computed control functions driving the HIV infection to a target steady state: (a) efficacy function for RTI $e_{R T I}(t)=$ $u_{1}(t)$, (b) efficacy function for PI $e_{P I}(t)=u_{2}(t)$.

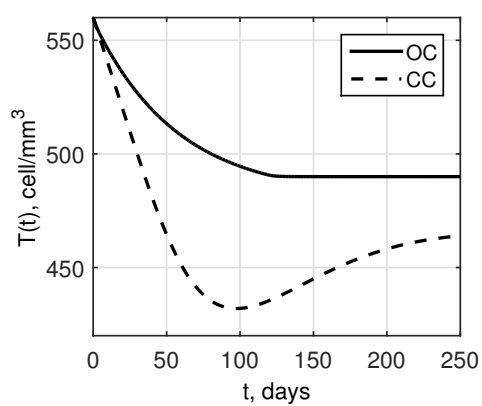

(a)

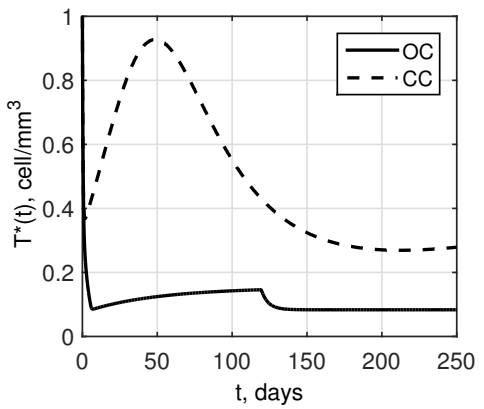

(b)

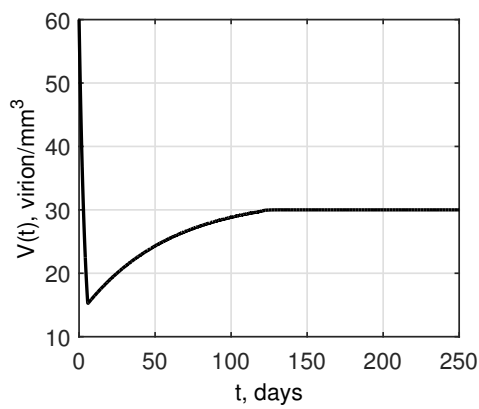

(c)

Figure 3. HIV infection dynamics for optimal- and time-constant control strategies: (a) uninfected CD4 ${ }^{+}$T-cells dynamics for optimal control (OC efficacy function) $T(t, u(t))$ and constant controls (CC) $\tilde{T}(t, A U C(u))$, (b) infected CD4 $4^{+}$T-cells and (c) viral load dynamics under optimal efficacy $T^{\star}(t, u(t)), V(t, u(t))$ and constant efficacy functions $\tilde{T}^{\star}(t, A U C(u)), \tilde{V}(t, A U C(u))$ regimes.

days rather than a stabilization at a steady sate level. The observed behaviours suggest that further analysis of the optimal drug dosage administration performed only for the $e_{\max }$ model will be sufficiently instructive.

To find the optimal doses of the ART drugs required to achieve the target concentrations specified by $C(t)$ we proceed with the orthogonal projection algorithm outlined in the previous section. To this end linear systems of equations with the Gram matrix for the set of basis functions $\Gamma_{i}(t)$ for every pharmacokinetic model specified in Table 2 are generated. By solving the system of equations we get the required values of $C_{0}^{\star}$ vector and the approximating drug kinetic profile $C_{\Gamma}(t)$.

Figure 5 gives an example of $C_{\Gamma}(t)$ behaviour during the first five days after initiation of the PI-type drug therapy. 


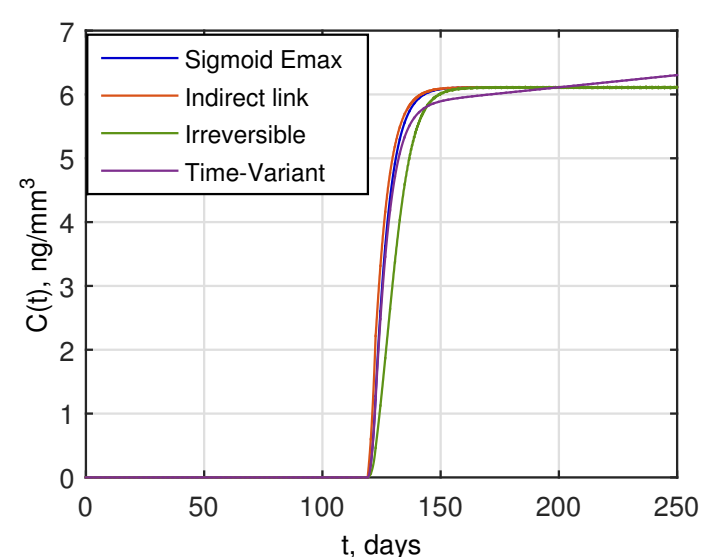

(a)

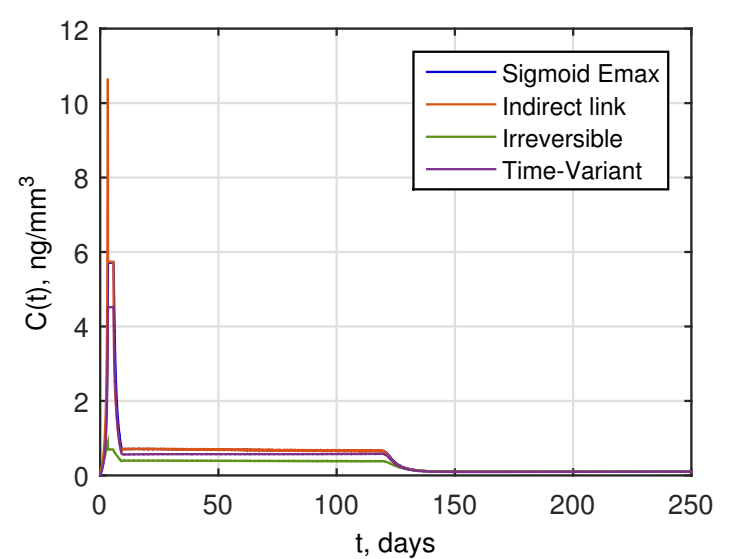

(b)

Figure 4. Concentration functions of antiviral drugs computed from four different pharmacodynamic models: (a) RTI-type drug, $C_{R T I}(t)=C\left(e_{R T I}(t)\right)$. (b) PI-type drug, $C_{P I}(t)=C\left(e_{P I}(t)\right)$.

Table 3. Approximation relative errors for RTI drug.

\begin{tabular}{lccccccc}
\hline Model type, $\boldsymbol{\tau}$ (hours) & $\mathbf{2}$ & $\mathbf{4}$ & $\mathbf{6}$ & $\mathbf{8}$ & $\mathbf{1 0}$ & $\mathbf{1 2}$ & $\mathbf{2 4}$ \\
\hline 1C & 0.0485 & 0.1079 & 0.1633 & 0.2164 & 0.2671 & 0.3151 & 0.5426 \\
CA & 0.0116 & 0.0342 & 0.0666 & 0.1077 & 0.1549 & 0.2057 & 0.4872 \\
C & 0.0477 & 0.1061 & 0.1605 & 0.2126 & 0.2621 & 0.3090 & 0.5306 \\
CA & 0.0158 & 0.0452 & 0.0841 & 0.1291 & 0.1767 & 0.2247 & 0.4706 \\
\hline
\end{tabular}

Table 4. Approximation relative errors for PI drug.

\begin{tabular}{lccccccc}
\hline Model type, $\boldsymbol{\tau}$ (hours) & $\mathbf{2}$ & $\mathbf{4}$ & $\mathbf{6}$ & $\mathbf{8}$ & $\mathbf{1 0}$ & $\mathbf{1 2}$ & $\mathbf{2 4}$ \\
\hline 1C & 0.0502 & 0.1113 & 0.1655 & 0.2215 & 0.2729 & 0.3247 & 0.5540 \\
1CA & 0.0121 & 0.0352 & 0.0690 & 0.1142 & 0.1632 & 0.2106 & 0.4885 \\
C & 0.0496 & 0.1100 & 0.1634 & 0.2186 & 0.2690 & 0.3200 & 0.5436 \\
2CA & 0.0135 & 0.0391 & 0.0743 & 0.1200 & 0.1666 & 0.2103 & 0.4549 \\
\hline
\end{tabular}

The behaviour of $C_{\Gamma}(t)$ for RTI of drug treatments is shown in Fig. 6. Here we consider one-compartment model with absorption and the time intervals between the drug intake ranging from 4 to 12 hours: $\tau=$ $\{4,8,12\}$.

Figures 5 and 6 also show the drug doses $C_{0}^{\star}$ administered sequentially at times $t_{i}=i \tau$ required to closely achieve the target concentration function $C(t)$. One can observe that the values of $C_{0}^{\star}$ are decreasing with smaller $\tau$ values. Indeed with shorter intervals between drug administrations larger quantities of the drug remain in the body and consequently the required drug amount to reach the target level becomes smaller. Obviously, the resulting drug concentration functions depend on the $\tau$ value and differ from one another. One can examine the problem of finding an optimal dosage interval $\tau$. The optimality can be addressed by considering an approximation criterion expressed as a relative error of the difference between the target drug concentration and the achievable concentration function in the Euclidean norm:

$$
R_{\text {err }}=\frac{\left\|\mathbf{C}-\mathbf{C}_{\Gamma}\right\|}{\|\mathbf{C}\|}, \quad \mathbf{C}=\left[C\left(t_{1}\right), C\left(t_{2}\right), \ldots, C\left(t_{N}\right)\right]^{T}, \quad t_{i} \in\left[0, T_{\text {cure }}\right], \quad i=1,, N .
$$

The relative approximation errors were computed for all pharmacokinetics models and values of $\tau$ ranging from 2 to 24 hours. The results are presented in Table 3 for RTI drug and Table 4 for PI drug. One can see that the optimal interval between drug administration is the one with a minimal error. The tables show that the best approximations for both drugs are obtained for the smallest $\tau$ values equal to two hours between intakes. The models with absorption provide a better approximation. Figure 7 depicts the drug concentration 


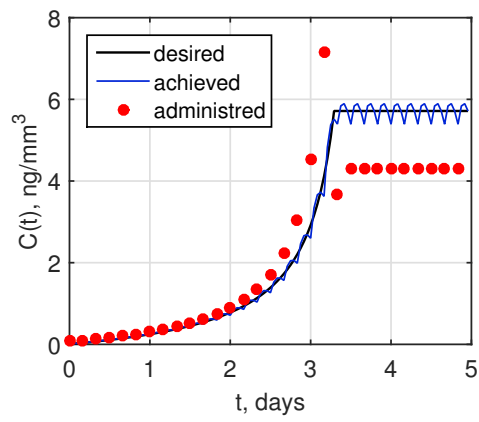

(a)

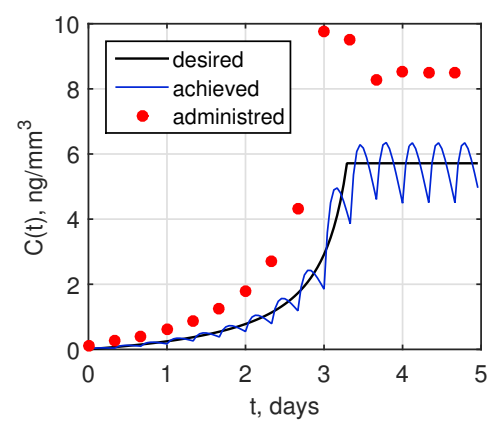

(b)

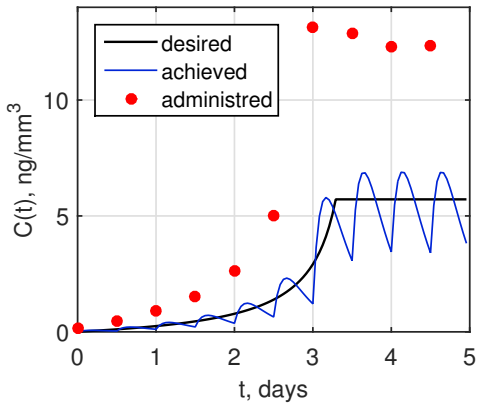

(c)

Figure 5. One-compartment model with absorption. The theoretical $C(t)$ and attainable $C_{\Gamma}(t)$ drug concentration kinetics for PI-type drug. The calculated medication doses $C_{0}^{\star}$ are shown by red circles for various intervals $(\tau)$ between the drug administration: (a) $\tau=4$ hours, (b) $\tau=8$ hours, (c) $\tau=12$ hours.

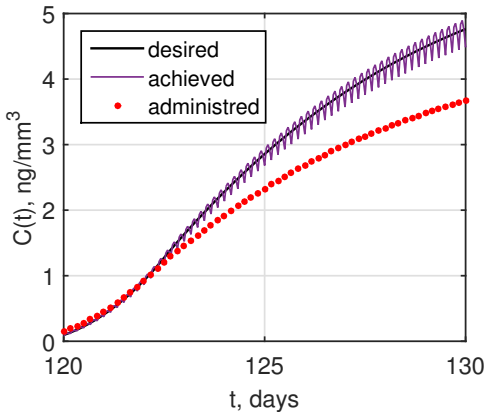

(a)

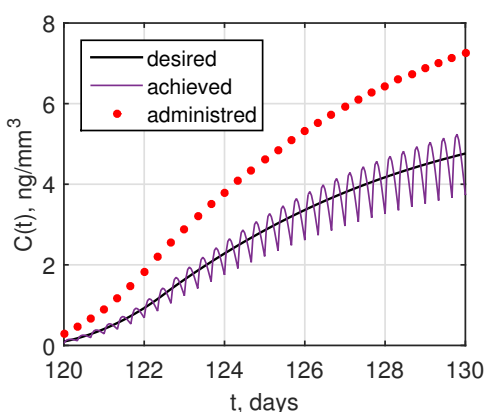

(b)

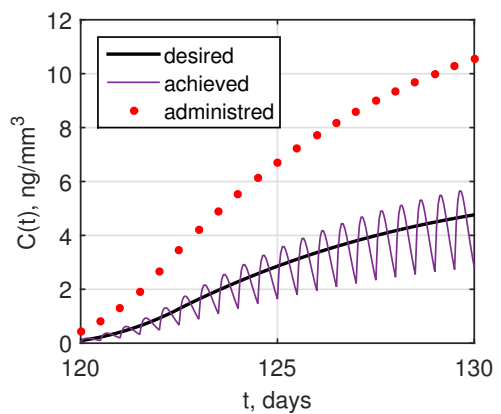

(c)

Figure 6. The concentration of the RTI-type drug suggested by the solution of the optimal control problem $C(t)$ and the best approximation to it attainable for the 'one-compartment with absorption' pharmacokinetics model $C_{\Gamma}(t)$. The estimated doses $C_{0}$ of the drug to be administered at the indicated times: (a) $\tau=4$ hours, (b) $\tau=8$ hours, (c) $\tau=12$ hours.

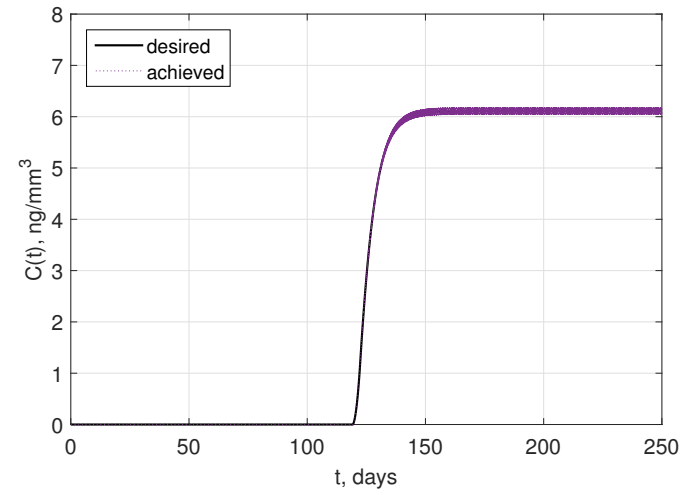

(a)

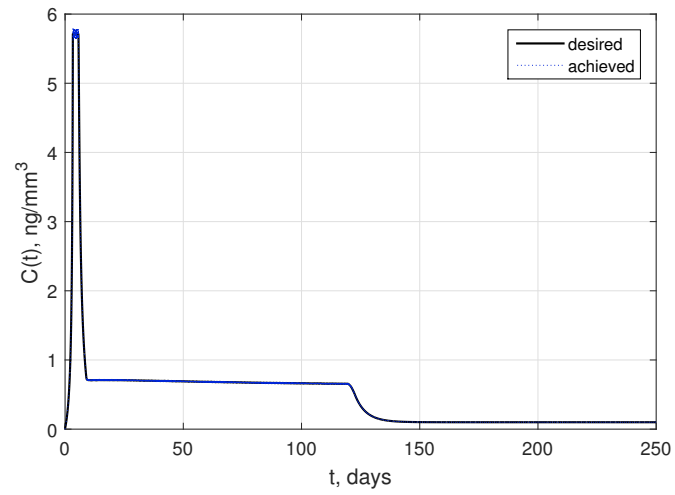

(b)

Figure 7. The long-term dynamics of the concentration of the RTI- (a) and PI-type (b) of the antiretroviral drugs suggested by the solution of the optimal control problem $C(t)$ and the best approximation to it attainable for the 'one-compartment with absorption' pharmacokinetics model $C_{\Gamma}(t)$ with a short-interval between the administration of the drug $\tau=2$ hours. 
functions for a long-term treatment for one-compartment model with absorption $\left(T_{\text {cure }}=250\right.$ (days)). One can see that the approximating concentration functions oscillate in the close vicinity of the target theoretical functions in relation to the basic drug kinetics model $\Gamma(t)$. The oscillations increase with increasing dosages $C_{0}$ or with time intervals $\tau$ between the drug intakes.

To verify the computed approximation $C_{\Gamma}(t)$ for the drug concentration function $C(t)$ we solved numerically the minimization problem (2.2) for the functional $Z\left(C_{0}\right)$ using MATLAB function 'fminunc' with zero initial guess vector. By finding the minimum values $\hat{C_{0}}=\arg \min \left(Z\left(C_{0}\right)\right)$ we evaluated $C_{\text {ver }}=\sum_{i=0}^{N} \hat{C_{0}^{\star}} \Gamma_{i}$ to be compared with the optimal projection based solution. The relative difference between the two functions $\left\|\mathbf{C}_{\Gamma}-\mathbf{C}_{\mathrm{ver}}\right\| /\left\|\mathbf{C}_{\Gamma}\right\|$ appears to be less than 0.0002 for all models and $\tau$ values used in the analysis and by two orders smaller than the relative approximation error.

\section{Discussion}

In the present study we examined the link between idealized control laws for the HIV infection obtained via the application of the optimal control theory to mathematical models of HIV infection dynamics. This goes beyond existing approaches towards computational modelling of HIV control which are based upon the assumption that the efficacy or control function can take any value at all times. A computational algorithm was developed that enables to determine the drug dosages $C_{0}$ that are required to achieve an optimal drug concentration $C(t)$ to have the efficacy $e(t)$ that stabilizes the viral load below a certain level $V(t) \leq \bar{V}$ and uninfected CD4 ${ }^{+}$T-cells above a certain level $T(t) \geq \bar{T}$. The algorithm approximates the effect of theoretical control functions by an optimal drug administration regime with the dose of the drug being an optimized parameter. The dependence on time intervals between the drug administration was explored. The developed algorithm has a modular structure so that the specific HIV infection dynamic model or the pharmacokinetics/dynamics model of the drugs can be replaced by refined ones. Our results indicate that in order to ensure a good approximation of the drug concentration kinetics suggested by the optimal control of the infection dynamics the intervals between the drug administration should be smaller and a two-fold reduction leads to a proportionate decrease in the relative error of the approximation. The pharmacokinetics models with absorption provide a better approximation to the target concentration profile than those without absorption.

Previous mathematical modelling studies on HIV dynamics and control considered the effect of drug treatment through an idealized 'efficacy' or control function term. In medical practice, the drug administration is constrained by the approved protocols and is based on prescription of standard doses of ART drugs in a regular manner [16]. In addition, the drug action is constrained by the pharmacokinetics and pharmacodynamics of the drug. Special clinical studies need to be conducted in order to identify the functions describing the drug efficacy and the kinetics of the drug in the body.

The design of patient-individual dosing regimes for HIV infection treatment remains a challenge. Further progress in this direction requires the parameterization of the side effects and the cost of the drugs, and considerations of multiscale regulation of virus infection and immune response dynamics. The first study along these lines was presented recently in [8]. Another important direction of analysis will be to explore the optimal drug administration regimen by varying both the dose and the timing between the interventions. Finally, our approach will be extended to consider the practically used therapies based on the combination of three and more antiviral drugs and to include immunomodulatory treatments.

Funding: The research was funded by the Russian Science Foundation (Grant No. 15-11-00029).

\section{References}

[1] B. M. Adams, H. T. Banks, M. Davidian, H. D. Kwon, H. T. Tran, S. N. Wynne, and E. S. Rosenberg, HIV dynamics: modelling, data analysis, and optimal treatment protocols. J. Comp. Appl. Math. 184 (2005), No. 1, 10-49. 
[2] V. N. Afanasiev, V. B. Kolmanovskii, and V. R. Nosov, Mathematical Theory for Construction of Control Systems. Vysshaya Shkola, Moscow, 1998, pp. 487-490 (in Russian).

[3] E. J. Arts and D. J. Hazuda, HIV-1 antiretroviral drug therapy. Cold Spring Harbor Perspectives in Medicine 2 (2012), No. 4, a007161.

[4] S. C. Beeler, H. T. Tran, and H. T. Banks, Feedback control methodologies for nonlinear systems. J. Opt. Theory Appl. 107 (2000), No. 1, 1-33.

[5] G. Bocharov, V. Chereshnev, I. Gainova, S. Bazhan, B. Bachmetyev, J. Argilaguet, J. Martinez, and A. Meyerhans, Human Immunodeficiency Virus Infection: from Biological Observations to Mechanistic Mathematical Modelling. Mathem. Modelling Natur. Phenom. 7 (2012), No. 5, 78-104.

[6] G. Bocharov, A. Kim, A. Krasovskii, V. Chereshnev, V. Glushenkova, and A. Ivanov, An extremal shift method for control of HIV infection dynamics. Russ. J. Numer. Anal. Math. Modelling 30 (2015), No. 1, 11-25.

[7] C. Fraser, T. D. Hollingsworth, R. Chapman, F. de Wolf, and W. P. Hanage, Variation in HIV-1 set-point viral load: epidemiological analysis and an evolutionary hypothesis. Proc. Natl. Acad. Sci. USA 104 (2007), No. 44,17441-6.

[8] M. M. Hadjiandreou, R. Conejeros, and I. Wilson, HIV treatment planning on a case-by-case basis. Int. J. Biol. Life Sci. 7 (2011), No. 3, 148-157.

[9] J. Lisziewicz, E. Rosenberg, J. Lieberman, et. al., Control of HIV despite the discontinuation of antiretroviral therapy. New England J. Medicine 340 (1999), No. 21, 1683-1683.

[10] G. Maartens, C. Celum, and S. R. Lewin, HIV infection: epidemiology, pathogenesis, treatment, and prevention. Lancet 384 (2014), 258-271.

[11] P. Macheras and A. Iliadis, Modelling in Biopharmaceutics, Pharmacokinetics and Pharmacodynamics Homogeneous and Heterogeneous Approaches. Springer, 2006, pp. 293-308.

[12] A. S. Perelson and P. N. Nelson, Mathematical analysis of HIV-1 dynamics in vivo. SIAM Review 41 (1999), No. 1, 3-44.

[13] A. S. Perelson and R. M. Ribeiro, Modelling the within-host dynamics of HIV infection. BMC Biology 11) (2013), 96.

[14] V. Radisavljevic-Gajic, Optimal control of HIV-virus dynamics. Annals Biomed. Engrg. 37 (2009), No. 6, 1251-1261.

[15] E. E. Tyrtyshnikov, Matrix Analysis and Linear Algebra. Physmathlit, Moscow, 2007, pp. 163-166 (in Russian).

[16] U. S. Department of Health and Human Services. Guidelines for the use of antiretroviral agents in HIV-infected adults and adolescents, (2003). URL:http://www.aidsinfo.nih.gov/guidelines.

[17] S. D. Varfolomeev and K. G. Gurevich, Biokinetics. Practical course. FAIR-PRESS, Moscow, 1999, $497-514$ (in Russian).

[18] L. H. Wang, G. E. Chittick, and J. A. McDowell, Single-dose pharmacokinetics and safety of abacavir (1592U89), zidovudine, and lamivudine administered alone and in combination in adults with human immunodeficiency virus infection. Antimicrobial Agents and Chemotherapy 43 (1999), No. 7, 1708-1715.

[19] A. Wong, The HIV pipeline. Nat Rev Drug Discov. 13 (2014), No. 9, 649-50.

[20] H. Wu, Y. Huang, E. P. Acosta, J. G. Park, S. Yu, S. L. Rosenkranz, and J. G. Gerber, Pharmacodynamics of antiretroviral agents in HIV-1 infected patients: using viral dynamic models that incorporate drug susceptibility and adherence. J. Pharmacokinetics Pharmacodynamics 33 (2006), No. 4, 399-419. 